\title{
A Comprehensive Review of the Prevention and Treatment of Heart Disease with a Plant-Based Diet
}

\author{
Stewart Rose* and Amanda Strombom \\ Plant-based diets in Medicine, USA \\ Submission: November 22, 2018; Published: December 14, 2018 \\ *Corresponding author: Stewart Rose, Plant-Based Diets in Medicine, 12819 SE 38th St, \#427, Bellevue, WA 98006, USA
}

\begin{abstract}
Epidemiological studies show that vegetarians have a much lower risk of myocardial infarction. Reductions of risk factors, and comorbidities such as angina, hypercholesterolemia, hypertension, diabetes, metabolic syndrome and obesity, have also been shown.

A low-fat plant-based diet can reverse or prevent further progression of coronary atheroma, improve endothelial dysfunction and is effective even in cases of severe stenosis. Studies show that in addition to regression, there is a remolding of the geometry of the stenosis with consequent improvement in coronary flow reserve.

Those following a plant-based diet have much lower total cholesterol and LDL. They also have lower levels of cardio-reactive protein, apolipoprotein (a) and apolipoprotein (b), plus levels of MPO, MMP-9, MMP-2 and MMP-9/TIMP-1 ratios. In addition, studies have determined that vegans produce less TMAO than their omnivorous counterparts after dietary challenge.

Long term exposure to persistent organic pollutants can drastically affect the circulatory system. The consumption of animal products is the greatest source of exposure to these toxins, due to bioaccumulation of these lipophilic toxins in animal tissues.

Interventional studies confirm that a plant-based diet is as effective in lowering cholesterol as statin drugs. Such studies also show that a plant-based diet can help treat heart failure and is very efficacious in treating angina pectoris. Vegetarians also show better improvements in cardiac rehab. Follow-up studies at one and four years confirm continued benefit to the patient, and patient compliance has been demonstrated over several years. Treatment with a plant-based diet is devoid of side effects and contraindications.

Keywords: Plant-based diet; Vegetarian; Vegan; Hypercholesterolemia; Angina; Stenosis; Heart disease; CVD; CAD; Persistent organic pollutants

Abbreviations: AhR: Aryl-Hydrocarbon Receptor; CABG: Coronary Artery Bypass Graft; CAD: Coronary Artery Disease; CHD: Coronary Heart Disease; CVD: Cardio-Vascular Disease; HD: Heart Disease; HDL: High Density Lipoprotein; LDL: Low Density Lipoprotein; MMP: Matrix Metalloproteinases; MPO: Myeloperoxidase; PAH: Polycyclic Aromatic Hydrocarbons; PCBs: Polychlorinated Biphenyls; POPs: Persistent Organic Pollutants; TCDD: Tetrachlorodibenzo-p-dioxin; TIMP: Tissue Inhibitors of Metalloproteinases; TMAO: Trimethylamine N-oxide; VLDL: Very Low Density Lipoprotein
\end{abstract}

\section{Introduction}

It has long been known from epidemiological studies that vegetarians have lower incidences of several common chronic diseases including ischemic heart disease. Epidemiological studies have also shown that they have lower incidences of risk factors for ischemic heart disease such as hypercholestrolemia, type 2 diabetes and essential hypertension.

This prompted research on using a vegetarian diet as a treatment for coronary artery disease. For over 45 years, evidence from interventional studies has strongly indicated that a low-fat plant-based diet is both safe and efficacious in the treatment of coronary artery disease (CAD). It's particularly effective in the treatment angina pectoris. Interventional studies have shown that a low-fat plant-based diet is a safe and efficacious alternative to other treatments.
This treatment can be used alone or in combination with standard treatment regimens, including medication, stenting and CABG. Treatment with the plant-based diet has the distinct advantages of having no adverse reactions or contraindications, is affordable, effectively treats common comorbidities and has been shown to have a high patient compliance.

\section{Reducing hypercholesterolemia}

Hypercholesterolemia is a well-known risk factor for CAD. Dietary saturated fat and cholesterol intake are shown to be strongly correlated with serum cholesterol levels. Less wellknown is the fact that both apolipoproteins (a) and (b) are significant risk factors for CAD. While statin drugs are effective at lowering serum cholesterol levels, they are not effective at lowering Apolipoprotein (a). 


\section{Epidemiology}

Vegetarians, and most especially vegans, have a much lower risk of hypercholesterolemia (for both total cholesterol and LDL) and a less atherogenic profile. They have been found to have lower total and LDL cholesterol levels on average. The following chart shows the average cholesterol levels among three dietary groups. $[1,2]$ Vegans, or total vegetarians, have the lowest levels (Figure $1)$.
The main reason for this is that animal products are high in both saturated fats and cholesterol. While saturated and trans-fat intake have the greatest effect on blood cholesterol concentrations, serum cholesterol concentrations also rise in response to dietary cholesterol intake. This relationship is linear within the range of cholesterol intakes in typical omnivorous diets, but at higher cholesterol intakes, the relationship is curvilinear; changes in dietary cholesterol have less impact on serum cholesterol [3].

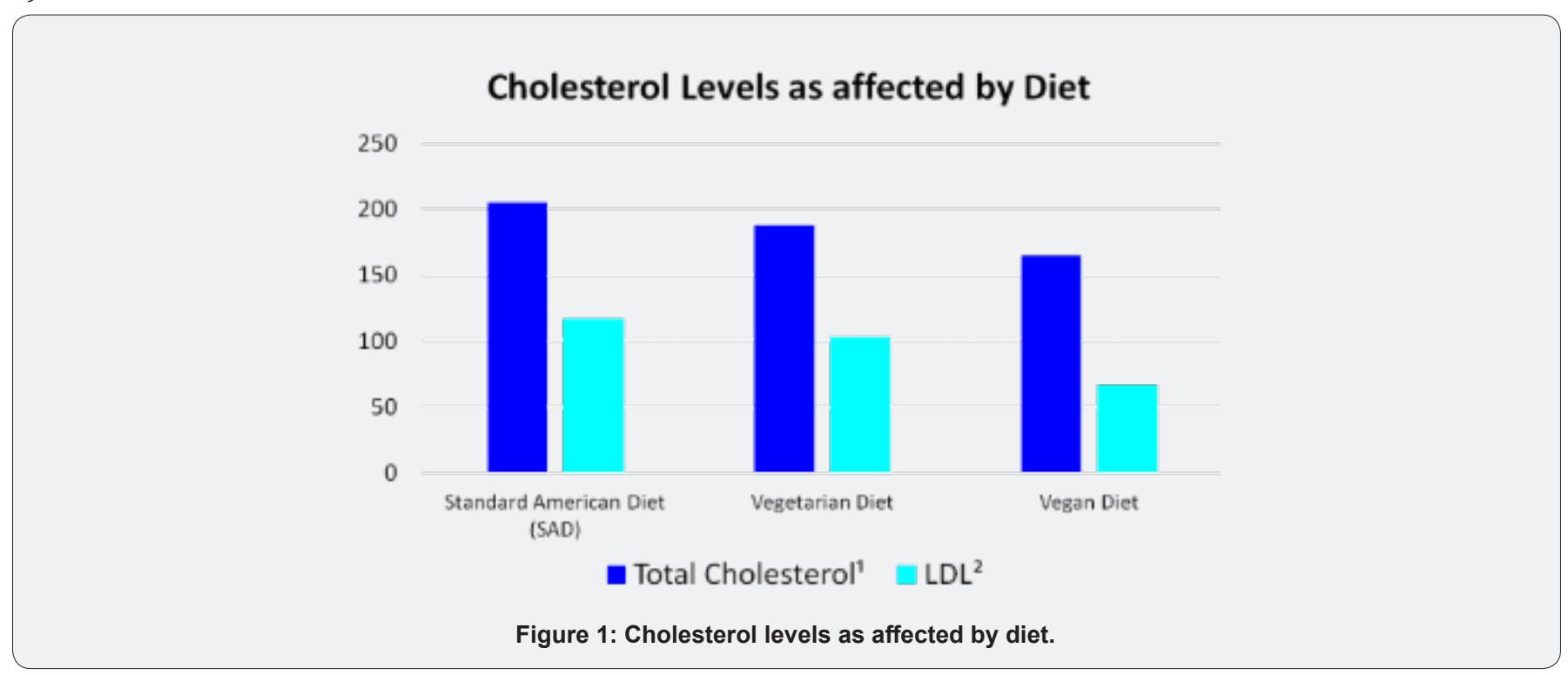

Another reason why low-fat vegetarian and vegan diets result in lower serum cholesterol levels is that they improve insulin sensitivity, which in turn can reduce cholesterol synthesis. This may account for a portion of the lower prevalence of hypercholesterolemia among vegetarians and vegans and for the results obtained in interventional studies [4,5].

The fact that vegetarians and especially vegans have lower cholesterol levels, and better cholesterol ratios, leads to much less atherosclerosis. One of the important mechanisms whereby LDL cholesterol results in atherosclerotic lesions involves the oxidation of LDL cholesterol [6,7]. There is evidence that the LDL cholesterol in vegetarians is much less oxidizable [8].

Vegans have also been found to have better regulation of the metabolism of triglyceride-rich lipoproteins than omnivores, because they are more efficient in removing remnants that are potentially atherogenic. In addition, the diminished cholesteryl ester transfer shown in the study, and the diminished LDL cholesterol levels that have been previously documented in other published studies, clearly suggest that a vegan diet offers some protection against atherogenesis [9]. A vegetarian diet also results in lower levels of C-reactive protein $[10,11]$.

In a cross-sectional study of apparently healthy vegetarian and non- vegetarian men, all over 35 years old, the vegetarian men had lower body mass index, systolic and diastolic blood pressures, fasting serum total cholesterol, LDL and non-HDLcholesterol, apolipoprotein B, glucose and glycated hemoglobin values in comparison with non- vegetarian men. The vegetarian men had better arterial function as measured by arterial stiffness determined by carotid-femoral pulse wave velocity, relative carotid distensibility and carotid intima-media thickness than non-vegetarian men [12].

\section{Apolipoproteins}

\section{Apolipoprotein (a)}

Several large population studies have shown a strong independent relationship between high apolipoprotein (a) (Lp(a)) levels and heart disease. This has led to the consensus agreement that it is a very important risk factor for cardiovascular disease, even when cholesterol levels and other classical risk factors such as elevated cholesterol, hypertension and diabetes have been taken into account. It is thought to increase the risk of cardiovascular disease by two different mechanisms:

1. It promotes atherosclerosis. Research studies have shown that Lp(a) may accelerate atherosclerotic damage. It is thought to increase the size of plaque atheroma in artery walls, causing inflammation, instability and growth of smooth muscle cells. It is retained in the artery wall more than LDL cholesterol as it binds to the artery lining through its "sticky" apolipoprotein.

2. It can trigger blockage of arteries by formation of clots. $\mathrm{Lp}(\mathrm{a})$ is thought to increase risk of heart attacks by interfering with clotting mechanisms and therefore promoting clot development on the inner surface of blood vessels. Lp(a) appears similar to proteins involved in clotting, such as 
plasminogen. It is thought to form a link between lipids and the coagulation system by preventing fibrinolysis.

Existing data show that elevated Lp(a) levels are associated with increased risk of coronary heart disease (CHD), stroke, peripheral arterial disease, and calcific aortic valve stenosis [1315]. In a recent individual-patient data meta-analysis of statintreated patients, elevated baseline and on-statin Lp(a) showed an independent approximately linear relation with cardiovascular disease risk. This study provides a rationale for lowering $\mathrm{Lp}(\mathrm{a})$ even in statin-treated patients [16].

Not only does a plant-based diet reduce serum cholesterol, but a recent study showed that $\mathrm{Lp}(\mathrm{a})$ could also be reduced. In a four-week study on patients following a plant-based diet, significant reductions were observed for serum $\mathrm{Lp}(\mathrm{a})$ with an average reduction of $-32.0 \mathrm{nmol} / \mathrm{L}$. Lp(a) had been considered resistant to change by lifestyle modification making this result very notable [17]. In contrast, initiation of statin therapy reduced LDL cholesterol (mean change $-39 \%$ [95\% CI -43 to -35$]$ ) without a significant change in Lp(a) [16].

\section{Apolilipoprotein (b)}

It is also evident that an increased serum apolipoprotein (b) (Lp(b)) concentration is an important CHD risk factor [18]. $\mathrm{Lp}(\mathrm{b})$ is the primary apolipoprotein of chylomicrons, VLDL, intermediate-density lipoprotein, and LDL particles. Prospective studies suggest that concentrations of $L p(b)$ are indicators of vascular heart disease and CVD risk [19,20].

Studies have also shown that vegetarians and vegans have lower $L p(b)$ than meat eaters [21]. Several studies show that a low-fat vegetarian diet reduces $L p(b)$ concentrations $[22,23]$. When a low-fat vegetarian diet is introduced, $L p(b)$ levels are reduced more than by other diets such the Atkins and South Beach diets [24].

\section{Hypercholesterolemia Intervention}

Vegetarian and vegan diets can be very efficacious in reducing serum cholesterol. Patients in a 4-week plant-based diet program had significant reductions in total cholesterol $(34 \mathrm{mg} / \mathrm{dl}), \mathrm{LDL}-\mathrm{C}$ (25 mg/dl), triglycerides (20mg/dl), hs-CRP (2.5 mg/dl), systolic BP (16 mmHg), and diastolic BP (9 mmHg) [25].

One study showed that a low-fat vegetarian diet was as effective at lowering serum cholesterol as the Standard Heart Association diet plus Lovostatin [26]. This study is notable because it contains nuts in the treatment regimen. (See Clinical Considerations for more information about the benefit of including nuts in the diet.).

Another study examining children and their adult parents found that a plant-based, or vegan, diet reduced total cholesterol, LDL cholesterol and C-reactive protein more than the American Heart Association diet. This study is especially important given the recent increase in the incidence of hypercholesterolemia in children and the fact that atherosclerosis seems to start early in life [27].

\section{Persistent Organic Pollutants}

\section{Epidemiology}

The increasing deterioration of the natural environment is having serious consequences on human health. The circulation system is the major organ exposed to xenobiotics and endobiotics during metabolic homeostasis [28-30] and exposure to persistent organic pollutants can drastically alter this system, resulting in cardiovascular diseases such as hypertension, atherosclerosis, and ischemic heart disease [30-36].

Exposure to persistent organic pollutants such as dioxins and dioxin-like polychlorinated biphenyls (PCBs) is associated with increased risk of multiple pro-inflammatory human diseases including diabetes, cancer, and cardiovascular disease [37-45].

High quality studies found consistent and significant doserelated increases in ischemic heart disease with dioxin [46]. Given the large worldwide burden of CAD, the potential role of dioxin exposure as a preventable risk factor could be of substantial public health and clinical interest [46].

\section{Mechanism A - The Aryl-Hydrocarbon Receptor}

The aryl-hydrocarbon receptor (AhR) is a well-known environmental sensor. Because many environmental pollutants contain exogenous AhR ligands, increasing attention is being given to the relationship between $\mathrm{AhR}$ and cardiovascular diseases. Recent evidence from gene knock-out studies and clinical trials suggests that not only does AhR have a major impact on general physiological functions, including immune responses, reproduction, oxidative stress, tumor promotion, the cell cycle, and proliferation, but also influences cardiovascular physiological functions [31-36].

$\mathrm{AhR}$ is a ligand activated transcription factor that mediates the cellular response to environmental contaminants, including dioxin and Polycyclic Aromatic Hydrocarbons (PAH) (from meat or cigarette smoke), and has recently been associated with CAD $[47,48]$. Exposure to pollutants containing ligands of AhR, such as dioxins, Tetrachlorodibenzo-p-dioxin (TCDD), PAH, and benzo( $\alpha$ ) pyrene, is thought to promote the development and progression of atherosclerosis, indicating that AhR may play a role in the regulation of atherosclerosis [37]. These environmental toxins, as well as endogenous activators such as ox-LDL, activate the AhR pathway, leading to increased inflammatory burden in the plaque [47].

Studies have shown that dioxin-like PCBs may also increase oxidative stress and subsequent chronic inflammatory states which can lead to glucose intolerance, alterations of lipid and cholesterol homeostasis, and other risk factors for multiple metabolic diseases [37,49].

\section{Mechanism B - TMAO}

Dioxin-like PCBs can lead to increased levels of the known proatherogenic nutrient biomarker Trimethylamine $\mathrm{N}$-oxide (TMAO) [50]. A strong link between plasma levels of nutrient-derived 
TMAO and coronary artery disease has been identified. Dietary precursors of TMAO include carnitine and phosphatidylcholine, which are abundant in animal-derived foods [50].

TMAO levels are strongly linked to human diseases, and plasma concentrations are correlated to dietary choices [51,52]. For example, diets high in red meat, and specifically L-carnitine, produce TMAO and accelerate atherosclerosis $[53,54]$. However, human studies have determined that vegans and vegetarians produce less TMAO than their omnivorous counterparts after dietary challenge [53].

\section{The Diet Connection}

Significant exposure of human populations to persistent organic pollutants (POPs) such as PCBs and dioxin occurs through consumption of fat-containing food such as fish, dairy products, and meat [55-57], with the highest POP concentrations being commonly found in fatty fish [55,56,58-62].

Humans bioaccumulate these lipophilic pollutants in their adipose tissues for many years because POPs are highly resistant to metabolic degradation $[57,63]$. This is likely to be one of the factors reducing the risk of CAD for vegans.

\section{Coronary Artery Disease}

\section{Epidemiology}

Epidemiological studies show a $40 \%$ risk reduction of ischemic heart disease [64] and a 50\% risk reduction of coronary heart disease mortality [65] for vegetarians.

\section{Pathophysiology}

Myeloperoxidase (MPO) is a leukocyte-derived pro-oxidant enzyme that is released from granules of neutrophils and monocytes [66]. MPO and its oxidant products, nitrotyrosine and chlorotyrosine, have been identified in atherosclerotic plaque and at the site of plaque rupture, and play an important role in the genesis of atherosclerosis [66]. MPO promotes a number of pathological events involved in plaque formation and rupture, including uptake of oxidized lipid by macrophages and impaired nitric oxide bioavailability. MPO levels independently predict outcomes in patients presenting with acute coronary syndromes or for evaluation of chest pain of suspected cardiac etiology and endothelial dysfunction [66].

Matrix Metalloproteinases (MMPs) are extracellular enzymes that are important in many physiologic and pathologic processes. Their activity is regulated mainly by tissue inhibitors of metalloproteinases (TIMPs). Their expression is associated with classical cardiovascular risk factors as well as with inflammation. They play a central role in atherosclerosis, plaque formation, platelet aggregation, acute coronary syndrome, restenosis, aortic aneurysms and peripheral vascular disease. Many studies have shown that commonly prescribed antihypertensive medications, glitazones and statins, may influence MMPs activity [67].
It is known that the arterial wall consists of collagen types I and III, macrophages and smooth muscle cells. The evolution of the atherosclerotic plaque from the fatty streak to advanced plaque is associated with an increase in its content of collagen [68], in the number of smooth muscle cells [69], and in MMP-9 levels [69]. Increased levels of MMP-9 are found more often in patients with unstable angina compared with those with stable angina [70]. Human coronary plaques that are less likely to rupture demonstrate lower MMP-9 expression [69]. In patients with coronary artery disease, higher MMP-9 levels are an independent risk factor of cardiovascular mortality [71]. Increased TIMP-1 levels have been reported consistently in human atherosclerotic plaques, mainly in relation to areas of calcification [72]. Increased circulating TIMP-1 levels have also been related to stable coronary [73], carotid [74], and peripheral artery atherosclerosis [74].

One study of circulating cardiovascular biomarker profiles compared the plasma concentrations of myeloperoxidase (MPO), matrix metalloproteinases MMP-9 and MMP-2, and tissue inhibitors of MMP TIMP-1 and TIMP-2, between healthy vegetarians and healthy omnivores. The study found significantly lower concentrations of MPO, MMP-9, MMP-2 and MMP-9/TIMP1 ratio in vegetarians compared to omnivores. Moreover, MMP-9 concentrations were correlated positively with leukocyte and neutrophil counts in both groups. Therefore, a vegetarian diet is associated with a healthier profile of cardiovascular biomarkers compared to omnivores [75].

E-selectin (cE-Selectin) is a cell adhesion molecule expressed only on endothelial cells activated by cytokines. Like other selectins, it plays an important role in inflammation [76].

The intercellular adhesion molecule-1 (cICAM-1) is an Ig-like cell adhesion molecule expressed by several cell types, including leukocytes and endothelial cells. It can be induced in a cell-specific manner by several cytokines, for example, tumor necrosis factor alpha, interleukin 1, and interferon gamma, and inhibited by glucocorticoids [77].

Upregulation of leukocyte adhesion molecules under atherogenic conditions is accompanied by the release of soluble forms of adhesion molecules into the bloodstream [78]. One study assessed the levels of circulating E-selectin (cE-selectin) and circulating intercellular adhesion molecule-1 (cICAM-1), in both vegetarians and subjects from the general population [78]. In this study vegetarians were characterized by a significantly lower cE-selectin levels. Vegetarians also showed a tendency towards lower cICAM-1 levels in comparison with control subjects [78]. Low cE-selectin levels of vegetarians may reflect the favorable cardiovascular risk profile of this group.

The lower levels of myeloperoxidase, matrix metalloproteinases and cE-selectin combine to give vegetarians a less atherogenic profile and helps explain their lower levels of atherosclerotic plaque. 


\section{General Intervention}

For over 45 years, evidence from interventional studies has strongly indicated that a low-fat plant-based diet is both safe and efficacious in the treatment of coronary artery disease (CAD). Researchers have investigated using a very low-fat vegan or nearly vegan diet to treat CAD of varying severity, and have achieved very positive results.

A moderately low-fat vegetarian diet was studied as an intervention for CAD as early as 1960. Morrison placed 50 patients with confirmed CAD on a moderately low-fat (25 g/day) vegetarian diet and followed them, and the 50 patients with CAD in the control group, for 12 years. While none of the patients in the control group survived for that length of time, $38 \%$ of the patients in the treatment group did [79]. It should be noted that since in 1960 neither stent, nor CABG surgery, nor cholesterol-reducing drugs, were available this was a very notable finding.

More recently in 1990, a prospective, randomized, controlled trial was done to determine whether comprehensive lifestyle changes affect coronary atherosclerosis after one year. Twentyeight patients were assigned to an experimental group (verylow-fat vegetarian diet, healthy lifestyle and stress management) and 20 to a standard care control group. 195 coronary artery lesions were analyzed by quantitative coronary angiography. The average percentage diameter stenosis regressed from an average $40.0 \%$ to $37.8 \%$ in the experimental group yet progressed from an average of $42.7 \%$ to $46.1 \%$ in the control group. When only lesions greater than $50 \%$ stenosed were analyzed, the average percentage diameter stenosis regressed from an average of $61.1 \%$ to $55.8 \%$ in the experimental group and progressed from an average of $61.7 \%$ to $64.4 \%$ in the control group. Overall, $82 \%$ of experimental-group patients had an average change towards regression [23]. In evaluating the regression, it is very important to keep in mind that blood flow increases by the radius raised to the $4^{\text {th }}$ power according to Poiseuille's Law, so small changes make a big difference.

This landmark study provided compelling evidence that a low-fat vegetarian diet can not only halt the progression of CAD, but even result in modest regressions in arterial stenosis. Given that CAD culminating in myocardial infarction is the leading cause of death in the developed world, and a tremendous burden on the health care system as well as on the patients themselves, the importance of this finding can hardly be overstated.

Following up on these results, researchers then looked to see if the treatment effects were sustained for longer periods of time, and if even further improvements could be obtained. The answer to both questions seems to be yes.

In a group of patients who participated in a 4-year follow up, the average percent diameter stenosis at baseline decreased 1.75 absolute percentage points after 1 year (a $4.5 \%$ relative improvement) and by 3.1 absolute percentage points after 4 years (a $7.9 \%$ relative improvement). In contrast, the average percent diameter stenosis in the control group increased by 2.3 percentage points after 1 year (a 5.4\% relative worsening) and by 11.8 percentage points after 4 years (a $27.7 \%$ relative worsening).

Patients in the experimental group lost $10.9 \mathrm{~kg}$ (23.9 lbs) at 1 year and sustained a weight loss of $5.8 \mathrm{~kg}(12.8 \mathrm{lbs})$ at 4 years, whereas weight in the control group changed little from baseline. In the experimental group, LDL cholesterol levels decreased by $40 \%$ at 1 year and remained $20 \%$ below baseline at 4 years. Experimental group patients also had a $91 \%$ reduction in reported frequency of angina after 1 year, and a $72 \%$ reduction after 4 years [80]. It is important to note that the results obtained above were dose dependent - the more closely patients adhered to the dietary regimen the better their results.

A smaller study also showed good results. 17 patients with CAD treated with a low-fat vegan diet, were followed for 5 years. Lesion analysis by percent stenosis showed that of 25 lesions, 11 regressed and 14 remained stable. Mean arterial stenosis decreased from an average of $53.4 \%$ to $46.2 \%$ [81].

A larger study, though with only a 3.7 year follow up, also showed positive results. 198 patients were placed on a lowfat vegan, or total vegetarian diet. $93 \%$ of patients experienced improvement or resolution of angina symptoms during the follow up period. Radiographic or stress testing results documented disease reversal in $22 \%$ of patients. $99.4 \%$ of adherent patients avoided major cardiac events. $89 \%$ patients were adherent to the treatment regimen. However, this was not a controlled study and the self-selected patients were very motivated [82].

An Indian study examined 360 coronary lesions in 123 such patients. Results were dose dependent. In CAD patients with the greatest adherence to a low-fat vegetarian diet, percent diameter stenosis regressed by an average of 18.23 absolute percentage points. $91 \%$ of all patients showed a trend towards regression, and $51.4 \%$ lesions regressed by more than 10 absolute percentage points [83].

A Dutch interventional study took patients who had at least one $50 \%$ obstruction and placed them on a vegetarian diet, although not as low in fat and cholesterol as other studies. After 2 years, $46 \%$ of patients showed no progression of the stenosis. Dietary changes were associated with a significant increase in linoleic acid content of cholesteryl esters, and a significant lowering of body weight, systolic blood pressure, serum total cholesterol, and the ratio of total to high-density lipoprotein (total/HDL) cholesterol [84].

\section{Coronary Perfusion Study}

As might be expected, patients on a low-fat vegetarian diet experience improvement in coronary perfusion as well. In one study after 4 years, the size and severity of perfusion abnormalities on dipyridamole PET images decreased after risk factor modification in the experimental group, compared with an increase of size and severity in controls. The percentage of left 
ventricle perfusion abnormalities, on the dipyridamole PET image of normalized counts outside 2.5 SDs of those of normal persons, worsened in controls by an average of $10.3 \%$ and improved in the experimental group by an average of $5.1 \%$. The percentage of left ventricle with activity less than $60 \%$ of the maximum activity worsened in controls by an average $13.5 \%$ and improved in the experimental group $4.2 \%$. The myocardial quadrant on the PET image with the lowest average activity, expressed as a percentage of maximum activity, worsened in controls by an average of $8.8 \%$ and improved in the experimental group by an average of $4.9 \%$. The size and severity of perfusion abnormalities on resting PET images were also significantly improved in the experimental group as compared with controls. The relative magnitude of changes in size and severity of PET perfusion abnormalities was comparable to, or greater than, the magnitude of changes in percent diameter stenosis, absolute stenosis lumen area, or stenosis flow reserve documented by quantitative coronary arteriography [85].

\section{Stenotic Morphology Study}

In 1992, an interesting study looked at the change of the geometric shape of the stenosis, in addition to the degree of stenosis, and their combined effect on flow reserve. Percent stenosis is an incomplete measure of stenosis because length, absolute lumen area and shape effects are not accounted for, and correlate poorly with the functional measure of coronary stenosis, coronary reserve flow [86]. Patients treated with a lowfat vegetarian diet show complex stenosis shape change, with profound effects on fluid dynamic severity, not accounted for by simple percent narrowing in a dose dependent manner. This effect is most pronounced with patients with severe pretreatment stenosis, with stenosis flow reserve less than 3. In this study, the minimal diameter increased by $18 \%$. Patients with a pretreatment average of $67 \%$ stenosis showed a $14 \%$ improvement in diameter [87]. As mentioned earlier, coronary blood flow effects are a function of arterial radius raised to the $4^{\text {th }}$ power, so small changes in the radius have proportionately much larger effects on flow capacity and functional severity of stenosis, thus contributing to the greater significance of stenosis flow reserve as a measure of change in severity.

\section{Angina pectoris}

An all-too-common comorbidity, angina, can also be treated with a low-fat plant-based diet. One study examined over 100 patients with CAD at 22 different clinics throughout the U.S. After 12 weeks, $74 \%$ of these patients placed on a plant-based diet were angina free, and an additional 9\% moved from limiting to mild angina [88]. Another study of patients placed on a vegetarian diet found that $91 \%$ of patients had a reduction in the frequency of angina episodes [80]. Using a purely vegan diet, one small study found complete remission of symptoms in all patients by the $6^{\text {th }}$ month [89].

\section{Heart failure}

Several epidemiological investigations have identified the following key risk factors for heart failure (HF): increasing age, hypertension, coronary artery disease, diabetes, obesity, valvular heart disease, and the metabolic syndrome [90].

Coronary artery disease, which can lead to heart failure, may be the underlying cause in most cases of heart failure patients with low ejection fraction. Coronary artery disease may also play a role in the progression of heart failure through mechanisms such as endothelial dysfunction, ischemia, and infarction, among others.

Since those following a plant-based diet are at lower risk of coronary artery disease, diabetes and obesity, they can be expected to be at lower of heart failure as well. Several population-based cohort studies that have demonstrated an inverse relationship between increased consumption of plant-based foods and incidence of heart failure [91-95].

Five prospective studies examined the association between meat consumption and HF incidence in separate medium to large, middle-aged cohorts. All of these studies found increased HF risk with meat consumption [96-100].

In a prospective cohort study of 21,275 participants from the Physicians' Health Study I, consumption of one egg a day increased the risk of heart failure by $28 \%$ and consuming two eggs a day increased the risk of heart failure by $64 \%$ [101]. In another prospective study of over 15,000 participants, those who ate a plant-based diet most of the time had a $42 \%$ reduced risk of heart failure [102].

The beneficial effects of a low-fat vegetarian diet are indicated for patients at risk of heart failure and who also have CAD. One study showed significant improvements in such patients with documented CHD, regardless of ejection fraction, in lifestyle behaviors, body weight, body fat, blood pressure, resting heart rate, total and LDL-cholesterol, exercise capacity, and quality of life by 3 months. Most improvements were maintained over 12 months [103].

A recent case report demonstrated the effects of a plant-based diet in a 79-year-old male with documented triple vessel disease (80-95\% stenosis) and left ventricular systolic dysfunction (ejection fraction 35\%) in the context of progressive dyspnea. Two months on a plant-based diet led to clinically significant reductions in body weight and lipids, with improved exercise tolerance and ejection fraction (+15\%) [95].

\section{Post Op Cardiac Rehabilitation Studies}

Researchers have also studied the effects of a low-fat vegetarian diet on patients who had already had standard treatments and were ready for post op cardiac rehabilitation.

One study compared patients in cardiac rehabilitation programs using either the standard treatment or a low-fat vegetarian diet (combined with stress reduction). Low-fat vegetarian program participants had significantly greater reductions in anginal frequency, body weight, body mass index, systolic blood pressure, total cholesterol, low-density lipoprotein cholesterol, glucose and dietary fat [104]. 
Another study looked at psychosocial risk factors and quality of life variables for patients in cardiac rehabilitation programs, using either the standard treatment or a low-fat vegetarian program. At 3 and 6 months, vegetarian participants demonstrated significant improvements in all 12 outcome measures, while the standard rehabilitation group improved in only 7 of the 12 [105].

\section{Clinical Considerations}

Substantial evidence indicates that plant-based diets can play an important role in preventing and treating CVD and its risk factors [106]. This suggests that a plant-based diet should be recommended as a prophylaxis to all patients, given that CAD is such a frequent cause of disability and death.

Dietary intervention is an extremely cost-effective treatment and may be the only viable treatment for those patients struggling with the affordability of other options. Some patients are either unwilling, fearful of or not good candidates for surgery. This treatment also offers them a nonsurgical option of proven efficacy.

One of the key advantages of the treatment of coronary heart disease with a low-fat vegetarian diet is the very low restenosis rate. One study reports the following average restenosis rates: balloon angioplasty $30-60 \%$, bare metal stents, $16-44 \%$, drug eluting stents $<10 \%$ [107]. Compare this with the low-fat vegan diet, which resulted in a zero-percentage restenosis rate in a study by Dean Ornish [108].

A low-fat vegetarian treatment regimen has also been shown suitable for diabetics with CAD. In a one-year study, diabetic patients with comorbid CAD showed good adherence to the treatment, and improvements in both cardiovascular and diabetic parameters, as demonstrated by significant improvements in weight, body fat, LDL cholesterol, and exercise capacity. About $20 \%$ of these patients were able to reduce or discontinue diabetic medications such as insulin or oral anti-hyperglycemics [109].

The problem of depression is a common concomitant of heart disease. A study using a low-fat plant-based diet in cardiac rehab patients, found that $80 \%$ saw very significant reductions in depression by 12 weeks, and the improvement was maintained for at least one year [110]. Another study of patients at high risk also showed an improvement in depressive symptoms [111].

While the interventional studies stressed a low-fat dietary regimen, there is good evidence that the inclusion of tree nuts, despite their fat content, reduces cardiac risk [112,113]. The research has been accumulating on the value of nuts in the prevention and treatment of a variety of diseases, including cardiovascular, indicating that the low-fat regimen now more commonly employed may be enhanced by moderate amounts of tree nuts. In one study, nut consumption was associated with reduced prevalence of high cholesterol and high blood pressure; having a history of heart attack, diabetes and gallstones; and markers of diet quality. In this cross-sectional analysis, higher nut consumption was also associated with lower body mass index and waist circumference [114].

\section{Fish Oil Supplementation}

There has been an unfortunate tendency amongst some physicians to recommend fish oils to their patients. However, this has not been borne out by the evidence. One metastudy conducted on the supposed benefits of fish oil reported, "All of the studies included were the gold-standard kind of clinical trial -- with people assigned at random to either take fish oil or a placebo. The studies ranged in length from one to nearly five years. The authors detected no reduction in any cardiovascular events, such as heart attacks, sudden death, angina, heart failures, strokes or death, no matter what dose of fish oil used [115]."

A recent meta-analysis of 10 randomized clinical trials also demonstrated that randomization to trial showed that fish oil had no significant effect on either of fatal CHD, nonfatal MI, stroke, revascularization events, or any major vascular events. Likewise, the study showed no significant association of omega-3 FA supplementation with all-cause mortality or cancer [116].

In patients with established cardiovascular disease or an increased risk of cardiovascular disease, omega-3 fatty acid supplementation also had no effect on major adverse cardiac events, all-cause mortality, sudden cardiac death, coronary artery revascularization, or hypertension [117]. In addition, a large, long-term randomised trial showed that fish oil supplements do not reduce the risk of cardiovascular events in patients with diabetes [118].

There has also been a mistaken notion that the Eskimo had a lower incidence of coronary heart disease, by virtue of their high fish oil consumption. This also turns out to not be the case. One report state, "Greenland Eskimos and the Canadian and Alaskan Inuit have CAD as often as the non-Eskimo populations [119]." Another study states, "Eskimos have CHD despite high consumption of omega-3 fatty acids." [120].

\section{Discussion}

\section{Treatment Advantages}

Interventional studies have shown that a low-fat $(<10 \%$ of calories) plant-based diet is a viable and highly advantageous alternative to other interventional strategies. The low-fat vegetarian diet also has no surgical risk of mortality, morbidity, no post op complications, and no adverse reactions or contraindications. The cost to the patient is minimal, and also both treats and lowers the risk of common comorbidities such as hypertension, diabetes and certain forms of cancer. It can serve as a monotherapy or as an adjunct to standard treatment regimens, including medication, stenting and CABG.

\section{Cost-Effective Treatment}

The treatment of CAD with a plant-based diet has been shown to be very cost effective. Highmark's Blue Cross estimated cost savings per participant in the Ornish low-fat vegetarian cardiac program is $\$ 16,186$ measured in 1999 dollars [121]. Estimated savings would likely be much higher today. A Mutual of Omaha Insurance study, also conducted in the 1990s, determined that 
for every dollar spent on the Ornish program there was a savings of $\$ 5.55$ in health care costs that would have otherwise accrued [122].

According to a Kaiser Family Foundation/New York Times survey, among people with health insurance, one in five $(20 \%)$ working age Americans report having had problems paying medical bills in the past year, often causing serious financial challenges and changes in employment. The situation is even worse among people who are uninsured or underinsured: half (53\%) face problems with medical bills, bringing the overall total to 26 percent [123]. Many people struggle with copayments and have high deductibles.

Coronary artery disease takes a tremendous toll in both lives and money. Heart disease remains the leading cause of death for both men and women [124]. CAD costs the United States $\$ 108.9$ billion each year $[125,126]$. Clearly, a more cost-effective treatment to Percutaneous Coronary Intervention and CABG is needed. As we have seen, treatment of CAD with a low-fat vegetarian diet would save a very considerable amount of money.

\section{A Needed Treatment Option}

As most physicians know, many patients these days attempt to gain health-related information and to treat themselves based upon what they read on the internet. Such information is often highly unreliable [127]. In our experience, most patients would rather get their health information and advice from their physicians, but turn to the internet when they can't. Therefore, to serve the best interests and needs of their patients, physicians should familiarize themselves with this treatment.

Research has documented the high rate of compliance with this treatment, especially when physicians explain the rationale behind the treatment and specifically prescribes it to their patients.

We live in an age of advanced medical technology. These advances have alleviated much suffering and saved countless lives. They have an unquestioned place in modern medicine. However, this can sometimes lead towards a kind of technological fundamentalism. Little notice is taken of treatments that, while lacking in technological sophistication, are nevertheless quite efficacious. This indeed seems to be the case with treating CAD with a low-fat vegetarian diet.

Fortunately, many doctors have already started to integrate therapeutic plant-based diets into their patients' prevention and treatment of CAD. The former president of the American College of Cardiology, Dr. Kim Williams, uses this modality of treatment for his patients [128].

In a recent article, he states:

"Unlike many of our cardiovascular prevention and treatment strategies, including antioxidants, vitamin E, folic acid and niacin to name a few, that have disintegrated over time, the "truth" (i.e., evidence) for the benefits of plant-based nutrition continues to mount. This now includes lower rates of stroke, hypertension, diabetes mellitus, obesity, myocardial infarction and mortality, as well as many non-cardiac issues that affect our patients in cardiology, ranging from cancer to a variety of inflammatory conditions. Challenges with the science are, however, less daunting to overcome than inertia, culture, habit and widespread marketing of unhealthy foods. Our goal must be to get data out to the medical community and the public where it can actually change livescreating healthier and longer ones [129]."

\section{Conflict of Interest}

The authors state no conflicts of interest.

\section{References}

1. Thorogood M, Carter R, Benfield L, McPherson K, Mann JI (1987) Plasma and lipoprotein cholesterol concentrations in people with different diets in Britain. Brit Med J (Clin Res Ed) 295(6594): 351-353.

2. Haddad E, Berk LS, Kettering JD, Hubbard RW, Peters WR (1999) Dietary intake and biochemical, hematologic, and immune status of vegans compared with nonvegetarians. Am J Clin Nutr 70 (3 Suppl): 586S-593S.

3. Hopkins PN (1992) Effects of dietary cholesterol on serum cholesterol: a meta-analysis and review. Am J Clin Nutr 55(6): 1060-1070.

4. Barnard ND, Cohen J, Jenkins DJ, Turner-McGrievy G, Gloede L, et.al. (2006) A low-fat vegan diet improves glycemic control and cardiovascular risk factors in a randomized clinical trial in individuals with type 2 diabetes. Diabetes Care 29(8): 1777-1783.

5. Tobin KA, Ulven SM, Schuster GU, Steineger HH, Andresen SM, et al. (2002) Liver X Receptors as Insulin-mediating Factors in Fatty Acid and Cholesterol Biosynthesis. J Biol Chem 277(12): 10691-10697.

6. Berliner JA, Navab M, Fogelman AM, Frank JS, Demer LL, et.al. (1995) Atherosclerosis: basic mechanisms. Oxidation, inflammation, and genetics. Circulation 91(9): 2488-2496.

7. Li D, Mehta JL (2005) Oxidized LDL, a critical factor in atherogenesis. Cardiovas Res 68(3): 353-354.

8. Lu SC, Wu WH, Lee CA, Chou HF, Lee HR, et al. (2000) LDL of Taiwanese vegetarians are less oxidizable than those of omnivores. J Nutr 130(6): 1591-1596.

9. Vinagre JC, Vinagre CG, Pozzi FS, Slywitch E, Maranhão RC (2013) Metabolism of triglyceride-rich lipoproteins and transfer of lipids to high-density lipoproteins (HDL) in vegan and omnivore subjects. Nutr Metab Cardiovas Dis 23(1): 61-67.

10. Krajcovicova-Kudlackova M, Blazicek P (2005) C-reactive protein and nutrition. Bratisl Lek Listy 106(11): 345-347.

11. Chen CW, Lin YL, Lin TK, Lin CT, Chen BC, et al. (2008) Total cardiovascular risk profile of Taiwanese vegetarians. Eur J Clin Nutr 62(1): 138-144.

12. Acosta-Navarro J, Antoniazzi L, Oki AM, Bonfim MC, Hong V, et al. (2017) Reduced subclinical carotid vascular disease and arterial stiffness in vegetarian men: The CARVOS Study. Int J Cardiol 230: 562-566.

13. Tsimikas S (2017) A test in context: lipoprotein(a): diagnosis, prognosis, controversies, and emerging therapies. J Am Coll Cardiol 69(6): 692-711.

14. Tsimikas S, Fazio S, Ferdinand KC, Ginsberg HN, Koschinsky ML, et al. (2018) NHLBI Working Group recommendations to reduce lipoprotein(a)-mediated risk of cardiovascular disease and aortic stenosis. J Am Coll Cardiol 71(2):177-192.

15. Yu B, Hafiane A, Thanassoulis G, Ott L, Filwood N, et al. (2017) Lipoprotein(a) induces human aortic valve interstitial cell calcification. JACC Basic Transi Sci 2(4): 358-371. 
16. Willeit P, Ridker PM, Nestel PJ, Simes J, Tonkin AM, et al. (2018) Baseline and on-statin treatment lipoprotein(a) levels for prediction of cardiovascular events: individual patient-data meta-analysis of statin outcome trials. Lancet 392(10155): 1311-1320.

17. Najjar RS, Moore CE, Montgomery BD (2018) Consumption of a defined, plant-based diet reduces lipoprotein(a), inflammation, and other atherogenic lipoproteins and particles within 4 weeks. Clin Cardiol 41(8): 1062-1068.

18. Contois JH, McConnell JP, Sethi AA, Csako G, Devaraj S, et al. (2009) Apolipoprotein B and Cardiovascular Disease Risk: Position Statement from the AACC Lipoproteins and Vascular Diseases Division Working Group on Best Practices. Clin Chem 55(3): 407-419.

19. Sandhu PK, Musaad SM, Remaley AT, Buehler SS, Strider S, et al. (2016) Lipoprotein Biomarkers and Risk of Cardiovascular Disease: A Laboratory Medicine Best Practices (LMBP) Systematic Review. J Appl Lab Med 1(2): 214-229.

20. Davidson MH, Ballantyne CM, Jacobson TA, Bittner VA, Braun LT, et al. (2011) Clinical utility of inflammatory markers and advanced lipoprotein testing: advice from an expert panel of lipid specialists. J Clin Lipidol 5(5): 338-367.

21. Bradbury KE, Crowe FL, Appleby PN, Schmidt JA, Travis RC, et al (2014) Serum concentrations of cholesterol, apolipoprotein A-I, and apolipoprotein B in a total of 1694 meat-eaters, fish-eaters, vegetarians, and vegans. Eur J Clin Nutr 68(2): 178-183.

22. Cooper RS, Goldberg RB, Trevisan M, Tsong Y, Liu K, et al. (1982) The selective lipid-lowering effect of vegetarianism on low density lipoproteins in a cross-over experiment. Atherosclerosis. 44(3): 293305

23. Ornish D, Brown SE, Scherwitz LW, Billings JH, Armstrong WT, et.al. (1990) Can lifestyle changes reverse coronary heart disease? The Lifestyle Heart Trial. Lancet 336(8708): 129-133.

24. Miller M, Beach V, Sorkin J, Mangano C, Dobmeier C, et al. (2009) Comparative effects of three popular diets on lipids, endothelial function, and C-reactive protein during weight maintenance. J Am Diet Assoc 109(4): 713-717.

25. Najjar RS, Moore CE, Montgomery BD (2018) A defined, plant-based diet utilized in an outpatient cardiovascular clinic effectively treats hypercholesterolemia and hypertension and reduces medications. Clin Cardiol 41(3): 307-313.

26. Jenkins DJ, Kendall CW, Marchie A, Faulkner DA, Wong JM, et al. (2005) Direct comparison of a dietary portfolio of cholesterol-lowering foods with a statin in hypercholesterolemic participants. Am J Clin Nutr 81(2): 380-387.

27. Macknin M, Kong T, Weier A, Worley S, Tang AS, et al. (2015) Plantbased, no-added-fat or American Heart Association diets: Impact on Cardiovascular Risk in Obese Children with Hypercholesterolemia and Their Parents. J Pediatr 166(4): 953-959.

28. Hanieh H (2014) Toward understanding the role of aryl hydrocarbon receptor in the immune system: current progress and future trends. BioMed Res Int p. 14.

29. Hernández-Ochoa I, Karman BN, Flaws JA (2009) The role of the aryl hydrocarbon receptor in the female reproductive system. Niochem Pharmacol 77(4): 547-559.

30. Yi T, Wang J, Zhu K, Tang Y, Huang S, et al. (2018) Aryl Hydrocarbon Receptor: A New Player of Pathogenesis and Therapy in Cardiovascular Diseases. Biomed Res Int. 2018: 6058784.

31. Xiao L, Zhang Z, Luo X (2014) Roles of xenobiotic receptors in vascular pathophysiology. Circ J 78(7): 1520-1530.

32. Chuang KJ, Yan YH, Chiu SY, Cheng TJ (2011) Long-term air pollution exposure and risk factors for cardiovascular diseases among the elderly in Taiwan. Occup Environ Med 68(1): 64-68.
33. Oesterling E, Toborek M, Hennig B (2008) Benzo[a]pyrene induces intercellular adhesion molecule-1 through a caveolae and aryl hydrocarbon receptor mediated pathway. Toxicol Appl Pharmacol 232(2): 309-316.

34. Kopf PG, Huwe JK, Walker MK (2008) Hypertension, cardiac hypertrophy, and impaired vascular relaxation induced by 2,3,7,8-Tetrachlorodibenzo-p-Dioxin are associated with increased superoxide. Cardiovasc Toxicol 8(4): 181-193.

35. Savouret JF, Berdeaux A, Casper RF (2003) The aryl hydrocarbon receptor and its xenobiotic ligands: A fundamental trigger for cardiovascular diseases. Nutr Metab Cardiovasc Dis 13(2): 104-113.

36. Dalton TP, Kerzee JK, Wang B, Miller M, Dieter MZ, et al. (2001) Dioxin exposure is an environmental risk factor for ischemic heart disease. Cardiovasc Toxicol 1(4): 285-298.

37. Perkins JT, Petriello MC, Newsome BJ, Hennig B (2016) Polychlorinated biphenyls and links to cardiovascular disease. Environ Sci Pollut Res Int 23(3): 2160-2172.

38. Kim SA, Kim KS, Lee YM, Jacobs DR, Lee DH (2015) Associations of organochlorine pesticides and polychlorinated biphenyls with total, cardiovascular, and cancer mortality in elders with differing fat mass. Environ Res 138: 1-7.

39. Pavuk M, Olson JR, Wattigney WA, Dutton ND, Sjödin A, et al. (2014) Predictors of serum polychlorinated biphenyl concentrations in Anniston residents. Sci Total Environ 496: 624-634.

40. Pavuk M, Olson JR, Sjödin A, Wolff P, Turner WE, et al. (2014) Serum concentrations of polychlorinated biphenyls (PCBs) in participants of the Anniston Community Health Survey. Sci Total Environ 473-474: 286-297.

41. Aminov Z, Haase RF, Pavuk M, Carpenter DO, Consortium AEHR (2013) Analysis of the effects of exposure to polychlorinated biphenyls and chlorinated pesticides on serum lipid levels in residents of Anniston, Alabama. Environ Health 12: 108.

42. Silverstone AE, Rosenbaum PF, Weinstock RS, Bartell SM, Foushee HR, et al. (2012) Polychlorinated biphenyl (PCB) exposure and diabetes: results from the Anniston Community Health Survey. Environ Health Perspect 120(5): 727-732.

43. Goncharov A, Bloom M, Pavuk M, Birman I, Carpenter DO (2010) Blood pressure and hypertension in relation to levels of serum polychlorinated biphenyls in residents of Anniston, Alabama. J Hypertens 28(10): 2053-2060.

44. Langer P, Kocan A, Tajtáková M, Susienková K, Rádiková Z, et al. (2009) Multiple adverse thyroid and metabolic health signs in the population from the area heavily polluted by organochlorine cocktail (PCB, DDE, HCB, dioxin). Thyroid Res 2(1): 3.

45. Lind PM, Orberg J, Edlund UB, Sjöblom L, Lind L (2004) The dioxinlike pollutant PCB 126 (3,3',4,4',5-pentachlorobiphenyl) affects risk factors for cardiovascular disease in female rats. Toxicol Lett 150(3): 293-299.

46. Humblet O, Birnbaum L, Rimm E, Mittleman M, Hauser R (2008) Dioxins and Cardiovascular Disease Mortality. Environ Health Perspect 116(11): 1443-1448.

47. Kim JB, Pjanic M, Nguyen T, Miller CL, Iyer D, et al. (2017) TCF21 and the environmental sensor aryl-hydrocarbon receptor cooperate to activate a pro-inflammatory gene expression program in coronary artery smooth muscle cells. PLoS Genet 13(5): e1006750.

48. Kim J, Pjanic M, Sazanova O, Wang T, Miller C, et al. (2018) TCF21 Regulates Coronary Artery Disease Causing Aryl-hydrocarbon Receptor Gene Expression and its Downstream Pathway Activation by Environmental Ligands. Circulation 132(Suppl 3).

49. Murphy M, Petriello M, Han S, Sunkara M, Morris A, et al. (2016) Exercise protects against PCB-induced inflammation and associated 
cardiovascular risk factors. Environ Sci Pollut Res Int. 23(3): 22012211.

50. Petriello M, Hoffman J, Sunkara M, Wahlang B, Perkins J, et al. (2016) Dioxin-like pollutants increase hepatic flavin containing monooxygenase (FMO3) expression to promote synthesis of the proatherogenic nutrient biomarker Trimethylamine $\mathrm{N}$-oxide from dietary precursors. J Nutr Biochem 33: 145-153.

51. Wang Z, Klipfell E, Bennett B, Koeth R, Levison B, et al. (2011) Gut flora metabolism of phosphatidylcholine promotes cardiovascular disease. Nature 472(7341): 57-63.

52. Fogelman A. (2015) TMAO is both a biomarker and a renal toxin. Circ Res 116(3): 396-397.

53. Koeth R, Wang Z, Levison B, Buffa J, Org E, et al. (2013) Intestinal microbiota metabolism of L-carnitine, a nutrient in red meat, promotes atherosclerosis. Nat Med 19(5): 576-585.

54. Ufnal M, Jazwiec R, Dadlez M, Drapala A, Sikora M, et al. (2014) Trimethylamine-N-oxide: a carnitine-derived metabolite that prolongs the hypertensive effect of angiotensin II in rats. Can J Cardiol 30(12): 1700-1705

55. Walker P, Rhubart-Berga P, McKenzie S, Kelling K, Lawrence R (2005) Public health implications of meat production and consumption. Pub Health Nutr 8(4): 348-356.

56. Sasamoto T, Ushio F, Kikutani N, Saitoh Y, Yamaki Y, et al. (2006) Estimation of 1999-2004 dietary daily intake of PCDDs, PCDFs and dioxin-like PCBs by a total diet study in metropolitan Tokyo, Japan. Chemosphere 64(4):634-641.

57. Fisher B. (1999) Most Unwanted. Environ Health Perspect 107(1): A18-23.

58. Bocio A, Domingo J (2005) Daily intake of polychlorinated dibenzo-pdioxins/polychlorinated dibenzofurans (PCDD/PCDFs) in foodstuffs consumed in Tarragona, Spain: a review of recent studies (2001-2003) on human PCDD/PCDF exposure through the diet. Environ Res 97(1) 1-9.

59. Schecter A, Colacino J, Haffner D, Patel K, Opel M, et al. (2010) Perfluorinated compounds, polychlorinated biphenyls, and organochlorine pesticide contamination in composite food samples from Dallas, Texas, USA. Environ Health Perspect 118(6): 796-802.

60. Darnerud P, Atuma S, Aune M, Bierselius R, Glynn A, et.al. (2006) Dietary intake estimations of organohalogen contaminants (dioxins, PCB, PBDE and chlorinated pesticides, e.g. DDT) based on Swedish market basket data. Food Chem Toxicol 44(9): 1597-1606.

61. Bergkvist C, Oberg M, Appelgren M, Becker W, Aune M, et al. (2008) Exposure to dioxin-like pollutants via different food commodities in Swedish children and young adults. Food Chem Toxicol 46(11): 33603367.

62. Dougherty C, Henricks Holtz S, Reinert J, Panyacosit L, Axelrad D, et al. (2000) Dietary exposures to food contaminants across the United States. Environ Res 84(2): 170-185.

63. Kiviranta H, Tuomisto JT, Tuomisto J, Tukiainen E, Vartiainen T (2005) Polychlorinated dibenzo-p-dioxins, dibenzofurans, and biphenyls in the general population in Finland. Chemosphere 60(7): 854-869.

64. Kwok C, Umar S, Myint P, Mamas M, Loke Y (2014) Vegetarian diet, Seventh Day Adventists and risk of cardiovascular mortality: a systematic review and meta-analysis. Int J Cardiol 176(3): 680-686.

65. Phillips R, Lemon F, Beeson W, Kuzma J (1978) Coronary heart disease mortality among Seventh-Day Adventists with differing dietary habits: a preliminary report. Am J Clin Nutr 31(10 Suppl): S191-S198.

66. Upadhyay R (2015) Emerging Risk Biomarkers in Cardiovascular Diseases and Disorders. J Lipids 2015: 971453.
67. Liu P, Sun M, Sader S (2009) Matrix metalloproteinases in cardiovascular diseases. Can J Cardiol 22 Suppl B:25B-30B.

68. Stary H, Chandler A, Dinsmore R, Fuster V, Glalov S, et al. (1995) A definition of advanced types of atherosclerotic lesions and a histological classification of atherosclerosis. A report from the Committee on Vascular Lesions of the Council on Arteriosclerosis, American Heart Association. Circulation 92(5): 1355-1374.

69. Brown D, Hibbs M, Kearney M, Isner J (1997) Differential expression of $92-\mathrm{kDa}$ gelatinase in primary atherosclerotic versus restenotic coronary lesions. Am J Cardiol 79(7): 878-882.

70. Brown D, Hibbs M, Kearney M, Loushin C, Isner JM (1995) Identification of $92-\mathrm{kD}$ gelatinase in human coronary atherosclerotic lesions: association of active enzyme synthesis with unstable angina. Circulation 91(8): 2125-2131.

71. Blankenberg S, Rupprecht H, Poirier O, Bickel C, Smieja M, et al. (2003) Plasma concentrations and genetic variation of matrix metalloproteinase 9 and prognosis of patients with cardiovascular disease. Circulation 107(12): 1579-1585.

72. Orbe J, Fernandez L, Rodríguez J, Rábago G, Belzunce M, et al. (2003) Different expression of MMPs/TIMP-1 in human atherosclerotic lesions. Relation to plaque features and vascular bed. Atherosclerosis 170(2): 269-276

73. Noji Y, Kajinami K, Kawashiri M, Todo Y, Horita T, et al. (2001) Circulating matrix metalloproteinases and their inhibitors in premature coronary atherosclerosis. Clin Chem Lab Med 39(5): 380-384.

74. Beaudeux J, Giral P, Bruckert E, Bernard M, Foglietti M, et al. (2003) Serum matrix metalloproteinase-3 and tissue inhibitor of metalloproteinases- 1 as potential markers of carotid atherosclerosis in infraclinical hyperlipidemia. Atherosclerosis 169(1): 139-146.

75. Navarro J, de Gouveia L, Rocha Penha L, Cinegaglia N, Belo V, et al. (2016) Reduced levels of potential circulating biomarkers of cardiovascular diseases in apparently healthy vegetarian men. Clin Chim Acta 461: 110-113.

76. Leeuwenberg J, Smeets E, Neefjes J, Shaffer M, Cinek T, et al. (1992) E-selectin and intercellular adhesion molecule-1 are released by activated human endothelial cells in vitro. Immunology 77(4): 543549.

77. van de Stolpe A, van der Saag P (1996) Intercellular adhesion molecule-1. J Mol Med (Berl) 74(1): 13-33.

78. Purschwitz K, Rassoul F, Reuter W, Purschwitz R, Jahn N, et al. (2001) Soluble leukocyte adhesion molecules in vegetarians of various ages. Z Gerontol Geriatr 34(6): 476-479.

79. Morrison LM (1960) Diet in Coronary Atherosclerosis. J Am Med Assoc 173: 884-888.

80. Ornish D, Scherwitz L, Billings J, Brown S, Gould K, et al. (1998) Intensive Lifestyle Changes for Reversal of Coronary Heart Disease. JAMA 280(23): 2001-2007.

81. Esselstyn CJ, Ellis S, Medendorp S, Crowe T (1995) A strategy to arrest and reverse coronary artery disease: a 5-year longitudinal study of a single physician's practice. J Fam Pract 41(6): 560-568.

82. Esselstyn CJ, Gendy G, Doyle J, Golubic M, Roisen M (2014) A way to reverse CAD? J Fam Pract 63(7): 356-364b.

83. Gupta S, Sawhney R, Rai L, Chavan V, Dani S, et al. (2011) Regression of coronary atherosclerosis through healthy lifestyle in coronary artery disease patients--Mount Abu Open Heart Trial. Indian Heart J 63(5): 461-469.

84. Arntzenius A, Kromhout D, Barth J, Reiber J, Bruschke A, et al. (1985) Diet, Lipoproteins, and the Progression of Coronary AtherosclerosisThe Leiden Intervention Trial. N Engl J Med 312: 805-811. 
85. Gould K, Ornish D, Scherwitz L, Brown S, Edens R, et al. (1995) Changes in myocardial perfusion abnormalities by positron emission tomography after long-term, intense risk factor modification. JAMA 274(11): 894-901.

86. Gould K (1990) Coronary arterial stenosis: a textbook of Coronary Pathophysiology, Quantitative Arteriography, Cardiac PET and reversal of Coronary Artery Disease. Elsevier, USA.

87. Gould K, Ornish D, Kirkeeide R, Brown S, Stuart Y, et al. (1992) Improved stenosis geometry by quantitative coronary arteriography after vigorous risk factor modification. Am J Cardiol 69(9): 845-853.

88. Frattaroli J, Weidner G, Merritt Worden T, Frenda S, Ornish D (2008) Angina pectoris and atherosclerotic risk factors in the multisite cardiac lifestyle intervention program. Am J Cardiol 101(7): 911-918.

89. Ellis F, Sanders T (1977) Angina and the Vegan Diet. American Heart Journal 93(6): 803-805

90. Velagaleti R, Vasan R (2007) Heart Failure in the 21st Century: Is it a Coronary Artery Disease Problem or Hypertension Problem? Cardiol Clin 25(4): 487-495.

91. Djoussé L, Driver J, Gaziano J (2009) Relation between modifiable lifestyle factors and lifetime risk of heart failure. JAMA 302(4): 394 400 .

92. Wang Y, Tuomilehto J, Jousilahti P, Antikainen R, Mähönen M, et al. (2011) Lifestyle factors in relation to heart failure among Finnish men and women. Circ Heart Fail 4(5): 607-612.

93. Pfister R, Sharp S, Luben R, Wareham N, Khaw K (2011) Plasma vitamin $\mathrm{C}$ predicts incident heart failure in men and women in European Prospective Investigation into Cancer and Nutrition-Norfolk prospective study. Am Heart J 162(2): 246-253.

94. Rautiainen S, Levitan E, Mittleman M, WolkA (2015) Fruit and vegetable intake and rate of heart failure: a population-based prospective cohort of women. Eur J Heart Fail 17(1): 20-26.

95. Choi E, Allen K, McDonnough M, Massera D, Ostfeld R (2017) A plantbased diet and heart failure: case report and literature review. J Geriatr Cardiol 14(5): 375-378.

96. Wirth J, di Giuseppe R, Boeing H, Weikert C (2016) A Mediterranean style diet, its components and the risk of heart failure: a prospective population-based study in a non-Mediterranean country. Eur J Clin Nutr 70(9): 1015-1021.

97. Nettleton J, Steffen L, Loehr L, Rosamond W, Folsom A (2008) Incident heart failure is associated with lower whole-grain intake and greater high-fat dairy and egg intake in the Atherosclerosis Risk in Communities (ARIC) study. J Am Diet Assoc 108(11): 1881-1887.

98. Ashaye A, Gaziano J, Djoussé L (2011) Red meat consumption and risk of heart failure in male physicians. Nutr Metab Cardiovasc Dis 21(12):941-946.

99. Kaluza J, Åkesson A, Wolk A (2015) Long-term processed and unprocessed red meat consumption and risk of heart failure: a prospective cohort study of women. Int J Cardiol 193: 42-46.

100. Kerley C (2018) A Review of Plant-based Diets to Prevent and Treat Heart Failure. Card Fail Rev 4(1): 54-61.

101. Djoussé L, Gaziano J (2008) Egg consumption and risk of heart failure in the Physicians' Health Study. Circulation 117(4): 512-516.

102. Lara K, Levitan E, Guitterrez O, Shikany J, Safford MJS, et al. (2017) Dietary patterns and incident heart failure in adults with no known coronary disease or heart failure: The REGARDS Cohort. Circulation 136(Suppl 1).

103. Pischke C, Weidner G, Elliott-Eller M, Ornish D (2007) Lifestyle changes and clinical profile in coronary heart disease patients with an ejection fraction of $40 \%$ in the Multicenter Lifestyle Demonstration Project.. Eur J Heart Fail 9(9): 928-934.

104. Aldana S, Whitmer W, Greenlaw R, Avins A, Slberg A, et al. (2003) Cardiovascular risk reductions associated with aggressive lifestyle modification and cardiac rehabilitation. Heart Lung 32(6): 374-382.

105. Aldana S, Whitmer W, Greenlaw R, Avins A, Thomas D, et al. (2006) Effect of intense lifestyle modification and cardiac rehabilitation on psychosocial cardiovascular disease risk factors and quality of life. Behav Modif 30(4): 507-525

106. Patel H, Chandra S, Alexander S, Soble J, Williams KS (2017) PlantBased Nutrition: An Essential Component of Cardiovascular Disease Prevention and Management. Curr Cardiol Rep. 19(10):104.

107. Buccheri D, Piraino D, Andolina G, Cortese B (2016) Understanding and managing in-stent restenosis: a review of clinical data, from pathogenesis to treatment. J Thorac Dis 8(10): E1150-E1162.

108. Ornish D (1998) Avoiding revascularization with lifestyle changes: The Multicenter Lifestyle Demonstration Project. Am J Cardiol 82(10B): 72T-76T.

109. Pischke C, Weidner G, Elliott-Eller M, Scherwitz L, Merritt-Worden T, et al. (2006) Comparison of coronary risk factors and quality of life in coronary artery disease patients with versus without diabetes mellitus. Am J Cardiol 97(9): 1267-1273.

110. Silberman A, Banthia R, Estay I, Kemp C, Studley J, et al. (2010) The effectiveness and efficacy of an intensive cardiac rehabilitation program in 24 sites. Am J Health Promot 24(4): 260-262.

111. Pischke C, Frenda S, Ornish D, Weidner G (2010) Lifestyle changes are related to reductions in depression in persons with elevated coronary risk factors. Psychol Health 25(9): 1077-1100.

112. Gopinath B, Flood V, Burlutksya G, Mitchell P (2015) Consumption of nuts and risk of total and cause-specific mortality over 15 years. Nutr Metab Cardiov Dis 25(12): 1125-1131.

113. Kris Etherton P (2014) Walnuts decrease risk of cardiovascular disease: a summary of efficacy and biologic mechanisms. J Nutr: 547S-554S.

114. Brown R, Gray A, Tey S, Chisholm A, Burley V, et al. (2017) Associations between Nut Consumption and Health Vary between Omnivores, Vegetarians, and Vegans. Nutrients 9(11): E1219.

115. Kwak S, Myung S, Lee Y, Seo H, Korean Meta-analysis Study Group (2012) Efficacy of Omega-3 Fatty Acid Supplements in the Secondary Prevention of Cardiovascular Disease. Arch Intern Med 172(9): 686694.

116. Aung T, Halsey J, Kromhout D, Gerstein H, Marchioli R, et al (2018) Associations of Omega-3 Fatty Acid Supplement Use With Cardiovascular Disease Risks: Meta-analysis of 10 Trials Involving 77917 Individuals. JAMA Cardiol 3(3): 225-234.

117. Rogers T, Seehusen D (2018) Omega-3 Fatty Acids and Cardiovascular Disease. Am Fam Physician 97(9): 562-564.

118. The ASCEND Study Collaborative Group (2018) Effects of n-3 Fatty Acid Supplements in Diabetes Mellitus. New Eng J Med 379: 1540 1550 .

119. Fodor J, Helis E, Yazdekhasti N, Vohnout B (2014) Fishing for the origins of the "Eskimos and heart disease" story: facts or wishful thinking? Can J Cardiol 30(8): 864-868.

120. Ebbesson S, Risica P, Ebbesson L, Kennish J (2005) Eskimos have CHD despite high consumption of omega-3 fatty acids: the Alaska Siberia project. Int J Circumpolar Health 64(4): 387-395

121. Highmark Blue Cross Blue Shield (1999) Two-Year Results of Highmark Blue Cross Blue Shield's Dr. Dean Ornish Program for 
Reversing Heart Disease Demonstrates Outstanding Lifestyle Improvements By The Men and Women Who Took Bold Step in Quest For Longer, Better, Happier Lives. PR Newswire, Pittsburgh, USA.

122. Ornish D (2010) Dr Dean Ornish's Program for Reversing Heart Disease. Random House. New York

123. Kaiser Family Foundation (2016) New Kaiser/New York Times Survey Finds One in Five Working-Age Americans With Health Insurance Report Problems Paying Medical Bills: https://www.kff org/health-costs/press-release/new-kaisernew-york-times-surveyfinds-one-in-five-working-age-americans-with-health-insurancereport-problems-paying-medical-bills /

124. Hasty RT, Garbalosa RC, Barbato VA, Valdes PJ Jr, Powers DW, et al. (2014) Wikipedia vs peer-reviewed medical literature for information about the 10 most costly medical conditions. J Am Osteop Assoc 114(5): 368-373.

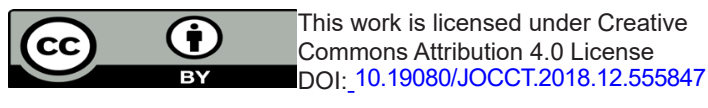

125. Centers for Disease Control and Prevention, National Center for Health Statistics (2015) Underlying Cause of Death 1999-2013: Wonder.CDC.gov/ucd-icd10.html.

126. Heidenreich P, Trogdon J, Khavjou O, Butler J, Dracup K, et al. (2011) Forecasting the future of cardiovascular disease in the United States: a policy statement from the American Heart Association. Circulation 123(8): 933-944.

127. Schwartz K, Roe T, Northrup J, Meza J, Seifeldin R, et al (2006) Family Medicine Patients' Use of the Internet for Health Information: A MetroNet Study. J Am Board Fam Med 19(1): 39-45.

128. Tuso P (2013) Nutritional Update for Physicians: Plant-Based Diets. Permanente J 17(2): 61-66.

129. Williams K (2017) Introduction to the "A plant-based diet and cardiovascular disease" special issue. J Geriatr Cardiol 14(5): 316.

Your next submission with Juniper Publishers
will reach you the below assets
- Quality Editorial service
- Swift Peer Review
- Reprints availability
- E-prints Service
- Manuscript Podcast for convenient understanding
- Global attainment for your research
- Manuscript accessibility in different formats
( Pdf, E-pub, Full Text, Audio)
- Unceasing customer service
Track the below URL for one-step submission
https://juniperpublishers.com/online-submission.php

